Pacific Journal of Mathematics

SEMI-DEVELOPABLE SPACES AND QUOTIENT IMAGES OF 


\title{
SEMI-DEVELOPABLE SPACES AND QUOTIENT IMAGES OF METRIC SPACES
}

\author{
Charles C. Alexander
}

In this paper semi-developable spaces are defined and, among $T_{0}$-spaces, are shown to be the same as the semimetrizable spaces. Strongly semi-developable spaces are defined in a natural way and proven to coincide with an important class of semi-metric spaces, namely those in which "Cauchy sequences suffice". These spaces are shown to possess several other interesting properties. Probably the most significant of these is that the strongly semi-develonable spaces are the hereditarily quotient Paimages of metric spaces. Other quotient images of metric sfaces are similarly characterized in terms of semi-developments.

1. Semi-deve'opable spaces. Until fairly recently there were almost no non-trivial topological restrictions on a space which guarantee the space to be semi-metrizable. Work along this line was initiated by Alexandrof and Niemytskii in their paper [1]. They proved that the developable spaces are precisely the spaces having a semi-metric satisfying the following condition. At each point there is a neighborhood of arbitrarily small diameter. Or equivalently, every convergent sequence is a Cauchy sequence. More recently Heath [10], Ceder [6], McAuley [13], and Arhangel'skii [3] have contributed new results in this area. Moreover, several authors have observed that there are numerous similarities between developable spaces and semi-metrizable spaces. This is especially true since so many theorems which hold for developable spaces are also valid for semi-metrizable spaces. In Theorem 1.3, the semi-metrizable spaces are shown to be the semi-developable $T_{0}$-spaces. This indicates the nature of the similarities between developable spaces and semi-metrizable spaces. Strongly semi-developable spaces are defined and, in Theorems 1.5 and 1.6, are shown to form a natural intermediate class of spaces between the semi-metrizable spaces and the developable spaces.

By a space we will mean a topological space as defined in [12]. All other definitions pertaining to topological spaces not specifically given in this paper are as found in [12].

Definition. A development for a space $X$ is a sequence

$$
\Delta=\left\{g_{n} \mid n=1,2, \cdots\right\}
$$

of open covers of $X$ such that $\left\{S t\left(x, g_{n}\right) \mid n=1,2, \cdots\right\}$ is a local base 
at $x$, for each $x \in X$. A space is developable if and only if there exists a development for the space.

Definitions. Let $\Delta=\left\{g_{n} \mid n=1,2, \cdots\right\}$ be a sequence of (not necessarily open) covers of a space $X$.

(i ) $\Delta$ is a semi-development for $X$ if and only if, for each $x \in X$, $\left\{\operatorname{St}\left(x, g_{n}\right) \mid n=1,2, \cdots\right\}$ is a local system of neighborhoods at $x$.

(ii) A semi-development $\Delta$ of $X$ is a strong semi-development if and only if for each $M \subset X$ and $x \in \bar{M}$ there exists a descending sequence $\left\{G_{n} \mid n=1,2, \cdots\right\}$ such that $x \in G_{n} \in g_{n}$ and $G_{n} \cap M \neq \varnothing$.

(iii) A semi-development $\Delta$ for $X$ is a point-finite semi-development if and only if for each $x \in X$ and for each positive integer $n, x$ is contained in only a finite number of sets in $g_{n}$.

A space $X$ is called semi-developable if and only if there exists a semi-development for $X$. Similarly, $X$ is called strongly (and/or point-finite) semi-developable if and only if there exists a strong (and/or point-finite) semi-development for $X$. We note that all of these properties are hereditary.

The following proposition clarifies the distinction between semidevelopment and strong semi-development.

Proposition 1.1. In order that a sequence $\Delta=\left\{g_{n} \mid n=1,2, \cdots\right\}$ of covers of a spase $X$ be a semi-development it is necessary and suffisient that for each $M \subset X$, and $x \in \bar{M}$ there exist a sequence $\left\{G_{n} \mid n=1,2, \cdots\right\}$ sush that $x \in G_{n} \in g_{n}$ and $G_{n} \cap M \neq \varnothing$.

\section{Proof. Trivial.}

Probably the first theorem concerning semi-developable spaces was the modified form of the Alexandrof-Urysohn Metrization Theorem given by Frink in [8]. It is stated here in terms of semi-developments.

THeOREM 1.2. A space $X$ is metrizable if and only if there exists a semi-development $\Delta=\left\{g_{n} \mid n=1,2, \cdots\right\}$ for $X$ sush that (a) $\cap\left\{S t\left(x, g_{n}\right) \mid n=1,2, \cdots\right\}=\{x\}$ for each $x \in X$, and (b) if $G, H \in g_{n}$, $n>1$, and $G \cap H \neq \varnothing$, then there is a set $J \in g_{n-1}$ sush that $G \cup H \subset J$.

A reasonable, but to our knowledge unconfirmed, conjecture is that condition (b) is exactly what is needed to guarantee the triangular property of the proposed metric. This is essentially the content of Theorem 1.3.

For later use, we note that every (point-finite and/or strongly) semi-developable space has a (point-finite and/or strong) semi-development $\left\{g_{n} \mid n=1,2, \cdots\right\}$ having the property that $g_{n+1}<g_{n}$ for each 
positive integer $n$. Hence, whenever the existence of a semi-development is assumed in a theorem, we may assume that it has the property mentioned above. Semi-developments having this property shall be called refining semi-developments.

Definition. A metric on a space $X$ is a function $d: X \times X \rightarrow R$ ( $R=$ real numbers) satisfying the following conditions:

For each $x, y, z \in X$ and $\varnothing \neq M \subset X$.

(i ) $d(x, x)=0$

(ii) $d(x, y)>0$, if $x \neq y$

(iii) $d(x, y)=d(y, x)$

(iv) $d(x, z) \leqq d(x, y)+d(y, z)$

(v) $x \in \bar{M}$ if and only if $d(x, M)=\inf \{d(x, m) \mid m \in M\}=0$.

Definition. A semi-metric on a space $X$ is a function $d: X \times X \rightarrow R$ satisfying conditions (i), (ii), (iii), and (v) above.

By a (semi-) metric space we mean a space $X$ together with a specific (semi-) metric on $X$. In this paper, whenever the (semi-) metric is not specified it will be assumed to be denoted by the letter $d$. The sphere about the point $x$ of radius $\varepsilon$ will be denoted by $S(x ; \varepsilon)$. Note that spheres need not be open but that $x \in \operatorname{Int} S(x ; \varepsilon)$ if $\varepsilon>0$.

It should be noted that in most of our theorems the $T_{0}$ property is assumed. This is usually done to insure that a semi-metric satisfies (ii) in the previous definition. Hence, in all such cases another theorem may be obtained by dropping the $T_{0}$ assumption and stating the theorem for a pseudo-metric space or a pseudo-semi-metric space, whichever the case may be.

Definition. Let $(X, d)$ be a semi-metric space. A sequence $\left\{x_{n} \mid n=1,2, \cdots\right\}$ in $X$ is a Cauchy sequence if and only if for each $\varepsilon>0$ there exists an integer $N$ such that $d\left(x_{n}, x_{m}\right)<\varepsilon$ whenever $m, n>N$.

Note that because of the lack of the triangle inequality not all convergent sequences in a semi-metric space are necessarily Cauchy sequences.

THEOREM 1.3. A space $X$ is semi-metrizable if and only if it is a semi-developable $T_{0}$-space.

Proof. Let $\Delta=\left\{g_{n} \mid n=1,2, \cdots\right\}$ be a refining s.d. for the $T_{0^{-}}$ space $X$ where, without loss of generality, $g_{1}=\{X\}$. For $x, y \in X$, let $n(x, y)$ denote the smallest integer $n$ such that there is no element of $g_{n}$ containing both $x$ and $y$. If no such integer exists let 
$n(x, y)=\infty$. Define $d: X \times X \rightarrow R$ as follows. For $x, y \in X$, let $d(x, y)=2^{-n(x, y)}$, where $2^{-\infty}=0$. Then clearly, for every $x, y \in X$, $d(x, x)=0$ and $d(x, y)=d(y, x)$. Also, if $x \neq y$, then, since $X$ is a $T_{0}$-space, there is an open set $U$ containing one of the points, say $x$, but not the other. Then there is an integer $n$ such that $x \in S t\left(x, g_{n}\right) \subset U$. Then $y \notin U$ implies $y \notin S t\left(x, g_{n}\right)$ which implies $y \notin S t\left(x, g_{i}\right)$ for each $i \geqq n$. It follows that $n(x, y) \leqq n$, and hence $d(x, y) \geqq 2^{-n}>0$.

Now note that $S\left(x ; 2^{-n}\right)=S t\left(x, g_{n}\right)$ for each $x \in X$ and each integer $n$. For $y \in S\left(x ; 2^{-n}\right)$ if and only if $d(x, y)<2^{-n}$ if and only if $n(x, y)>n$ if and only if there exists $G \in g_{n}$ such that $x, y \in G$ if and only if $y \in S t\left(x, g_{n}\right)$. Now let $M \subset X$. Then $x \in \bar{M}$ if and only if $S t\left(x, g_{n}\right) \cap M \neq \varnothing$ for each integer $n$ if and only if $S\left(x ; 2^{-n}\right) \cap M \neq \varnothing$ for each integer $n$ if and only if $d(x, M)=0$. Hence, $d$ is a semimetric on $X$.

Conversely, assume that $d$ is a semi-metric on $X$. For each positive integer $n$, let $g_{n}$ be the collection of all sets of diameter less than $1 / n$. Then for each $n, S(x ; 1 / n)=S t\left(x, g_{n}\right)$. For let $y \in S(x ; 1 / n)$. Then $G=\{x, y\} \in g_{n}$ implies $y \in S t\left(x, g_{n}\right)$. On the other hand, let $y \in S t\left(x, g_{n}\right)$. Then there is $G \in g_{n}$ such that $x, y \in G$, and therefore, $d(x, y) \leqq \operatorname{diam} G<1 / n$. Thus, $y \in S(x ; 1 / n)$.

Now let $U$ be an open set containing the point $x$. Then there is an integer $n$ such that $x \in \operatorname{Int} S(x ; 1 / n) \subset S(x ; 1 / n) \subset U$. Therefore, $x \in \operatorname{Int} S t\left(x, g_{n}\right) \subset S t\left(x, g_{n}\right) \subset U$. Hence $\left\{g_{n} \mid n=1,2, \cdots\right\}$ is a semidevelopment for $X$.

Corollary 1.4. Every $T_{0}$ semi-developable spase is $T_{1}$.

Notation. Given a semi-development $\Delta$ for a $T_{0}$-space $X$, we will let $d_{\Delta}$ denote the semi-metric on $X$ defined from $\Delta$ as above. Similarly, given a semi-metric $d$ on $X$, we will let $\Delta_{d}$ denote the semi-development on $X$ defined from $d$ as above.

THEOREM 1.5. In a semi-metris spase the following conditions are equivalent:

(1) For eash $M \subset X$ and each $x \in \bar{M}$, there exists a descending sequence of sets $\left\{G_{n} \mid n=1,2, \cdots\right\}$ of arbitrarily small diameters sush that for each $n, x \in G_{n}$ and $x \in G_{n} \cap M \neq \varnothing$.

(2) For eash $M \subset X$ and eash $x \in \bar{M}$, there exists a Caushy sequense in $M$ converging to $x$.

(3) Every convergent sequence has a Caushy subsequense.

Proof. Let $d$ be a semi-metric on $X$.

(1) implies (3). Let $S=\left\{x_{n} \mid n=1,2, \cdots\right\}$ be a sequence in $X$ 
converging to the point $x \in X$. If $x_{n}=x$ for infinitely many $n$, then clearly we can define a Cauchy subsequence of $S$. Otherwise let $M=\left\{x_{n} \mid n=1,2, \cdots\right\} \backslash\{x\}$. Then $x \in \bar{M}$ implies, by (1), that there is a descending sequence of sets $\left\{G_{n} \mid n=1,2, \cdots\right\}$ of arbitrarily small diameters such that for each $n, x \in G_{n}$ and $G_{n} \cap M \neq \varnothing$. We now define a subsequence of $\left\{x_{n} \mid n=1,2, \cdots\right\}$ inductively. Choose $x_{n_{1}} \in G_{1} \cap M$. Suppose $x_{n_{i}}$ has been chosen for each $i=1,2, \cdots, k-1$, such that $x_{n_{i}} \in G_{i} \cap M$ and $n_{i}>n_{i-1}$. Now observe that $G_{k} \cap M$ is infinite. For suppose not; say $G_{k} \cap M=\left\{a_{1}, \cdots, a_{m}\right\}$. Then there exists $n_{0}>k$ such that diam $G_{n_{0}}<\min \left\{d\left(x, a_{i}\right) \mid i=1,2, \cdots, m\right\}$. Clearly $a_{i} \notin G_{n_{0}}$ for each $i=1,2, \cdots, m$. But then

$$
M \cap G_{n_{0}} \subset M \cap G_{k}=\left\{a_{1}, \cdots, a_{m}\right\}
$$

implies $M \cap G_{n_{0}}=\varnothing$, which is a contradiction. Hence we can choose $x_{n_{k}} \in G_{k} \cap M$ such that $n_{k}>n_{k-1}$. Thus we have defined a subsequence $\left\{x_{n_{k}} \mid k=1,2, \cdots\right\}$ of $S$ which is Cauchy. For let $\varepsilon>0$ be given. Then there is an integer $N$ such that diam $G_{N}<\varepsilon$. For $i, j \geqq N$ we then have $x_{n_{i}} \in G_{i} \subset G_{N}$ and $x_{n_{j}} \in G_{j} \subset G_{N}$. Thus $d\left(x_{n_{i}}, x_{n_{j}}\right) \leqq$ diam $G_{N}<\varepsilon$.

(3) implies (2): Assume $M \subset X$ and $x \in \bar{M}$. Since $X$ is first countable there is a sequence $\left\{x_{n} \mid n=1,2, \cdots\right\}$ in $M$ which converges to $x$. By (3), this sequence has a Cauchy subsequence $\left\{x_{n_{k}} \mid k=1,2, \cdots\right\}$. Then $\left\{x_{n_{k}} \mid k=1,2, \cdots\right\}$ is a Cauchy sequence in $M$ converging to $x$.

(2) implies (1): Let $M \subset X$ and assume $x \in \bar{M}$. Then, by (2), there is a Cauchy sequence $\left\{x_{n} \mid n=1,2, \cdots\right\}$ in $M$ which converges to $x$. For each $n$, let $G_{n}=\left\{x_{i} \mid i \geqq n\right\} \cup\{x\}$. Then $\left\{G_{n} \mid n=1,2, \cdots\right\}$ is a descending sequence of sets of arbitrarily small diameters such that for each $n, x \in G_{n}$ and $G_{n} \cap M \neq \varnothing$.

Definition. A space $X$ is strongly semi-metrizable if and only if a semi-metric satisfying any one of the conditions of the previous theorem can be realized on $X$. Such a semi-metric is called a strong semi-metric.

Theorem 1.6. A space $X$ is strongly semi-metrizable if and only if it is a strongly semi-developable $T_{0}$-space.

Proof. Let $d$ be a strong semi-metric for $X$. Then, by Theorem 1.5, $d$ satisfies condition (1). Now consider the semi-development $\Delta_{d}$ defined in Theorem 1.3. By the definition of $\Delta_{d}$ and the fact that $d$ satisfies the condition (1), it follows immediately that $\Delta_{d}$ is a strong semi-development.

Conversely, let $\Delta=\left\{g_{n} \mid=1,2, \cdots\right\}$ be a refining strong semi- 
development for the $T_{0}$-space $X$. Let $d_{\Delta}$ be the semi-metric on $X$ as defined in Theorem 1.3. Observe that with this semi-metric, diam $G \leqq 2^{-n}$ for each $G \in g_{n}$ and $n=1,2, \cdots$. Thus it follows from the definition of a strong semi-development that $d_{\Delta}$ satisfies condition (1) of the previous theorem and hence all of the conditions.

In [13], example 3.2 exhibits a paracompact, hereditarily separable semi-metric space which is not developable. It is easy to see that the semi-metric defined there is a strong one. This shows that a strongly semi-developed space need not be developable.

In [5], Morton Brown noted the open question: "Does every semi-metrizable space have a semi-metric under which all spheres are open?" He then mentioned that such semi-metric spaces have the property that every convergent sequence has a Cauchy subsequence, i.e., are strongly semi-metrizable. R. W. Heath answered Brown's question negatively in [9]. However in doing so he implicitly raised two questions: (1) Does every strongly semi-metrizable space have a semi-metric under which all spheres are open, and (2) Is every semimetrizable space strongly semi-metrizable? It can be shown that Heath's space in [9] is strongly semi-metrizable and hence serves to supply a negative answer to (1). The answer to (2) remains unknown. Applying Theorems 1.5 and 1.6, we see that (2) is equivalent to the following question: Is every semi-developable $T_{0}$-space strongly semidevelopable?

The Urysohn Metrization Theorem [12; page 125] states that a regular $T_{1}$-space with a countable base is metrizable. However it is not true that every separable regular $T_{1}$-space is metrizable. Indeed the Example 3.2 in [13] mentioned above exhibits a paracompact, hereditarily separable, semi-metrizable space which is not metrizable. It is in this light that the following theorem is of interest.

TheOREM 1.7. Every separable regular $T_{0}$-space with a pointfinite semi-development is metrizable.

Proof. Let $X$ be a separable regular $T_{0}$-space and let

$$
\Delta=\left\{g_{n} \mid n=1,2, \cdots\right\}
$$

be a point-finite semi-development for $X$. Let $Q$ be a countable dense subset of $X$. Since, by Corollary $1.4, X$ is a regular $T_{1}$-space, it suffices to show that $X$ has a countable base, by the Urysohn Metrization Theorem.

Let $S=\left\{G \mid q \in G \in g_{k}\right.$ for some $q \in Q$ and some integer $\left.k\right\}$. Then $S$ is countable since each point of the countable set $Q$ is contained in only countably many sets of the semi-development $\Delta$. Thus it suffices 
to show that the interiors of the closures of finite unions of members of $S$ is a base for $X$.

Let $U$ be an open set in $X$ containing the point $x \in X$. Since $X$ is regular, there is an open set $V$ such that $x \in \bar{V} \subset U$. Define a subcollection $S^{\prime}$ of $S$ as follows:

$$
S^{\prime}=\{G \in S \mid x \in G \subset V\} .
$$

Now $S^{\prime} \neq \varnothing$. For $x \in V$ and $V$ is open implies there is an integer $N$ such that $x \in \operatorname{Int} S t\left(x, g_{N}\right) \subset S t\left(x, g_{N}\right) \subset V$. Since $Q$ is dense in $X$, $\operatorname{St}\left(x, g_{N}\right) \cap Q \neq \varnothing$. Hence, there is an element $q \in Q$ and $G \in g_{N}$ such that $x, q \in G$ and $G \subset V$. Then clearly $G \in S^{\prime}$

Let $S^{\prime}=\left\{G_{1}, G_{2}, \cdots\right\}$ be an enumeration of $S^{\prime}$. For each $n$, let $T_{n}=\cup\left\{G_{i} \mid i=1, \cdots, n\right\}$. Now let $\left\{W_{n} \mid n=1,2, \cdots\right\}$ be an open local base at $x$ such that for each $n, W_{n+1} \subset W_{n} \subset V$. It suffices to show that for some $n_{0}, W_{n_{0}} \subset \overline{T_{n_{0}}}$. For in that case $x \in \operatorname{Int} \overline{T_{n_{0}}} \subset \bar{V} \subset U$ and Int $\bar{T}_{n_{0}}$ is a member of the proposed base. Suppose that this is not the case, i.e., that for each integer $n, W_{n} \backslash \overline{T_{n}} \neq \varnothing$. Then for each $n$, since $W_{n} \backslash \overline{T_{n}}$ is an open set and $Q$ is dense, there exists

$$
x_{n} \in\left(W_{n} \backslash \overline{T_{n}}\right) \cap Q .
$$

Then the sequence $\left\{x_{n} \mid n=1,2, \cdots\right\}$ so defined clearly converges to $x$. Observe that for each $n, x_{n} \in T_{n}$ and the sets $\left\{T_{k} \mid k=1,2, \cdots\right\}$ are ascending. Thus $x_{n} \notin T_{k}$ for each $k \leqq n$.

Now let $N$ be an integer chosen as before such that $S t\left(x, g_{N}\right) \subset V$. Notice that the only way elements of $g_{N}$ which contribute to $S t\left(x, g_{N}\right)$ might be excluded from $S^{\prime}$ is by not containing an element of $Q$. As was seen before there is at least one element of $g_{N}$ which belongs to $S^{\prime}$. Since there are only finitely many such elements there is one which has a larger index in the enumeration of $S^{\prime}$ than any of the others. If $n_{0}$ is the index of that element, we see that $T_{n_{0}}$ contains the union of these elements. By a comment above, for $n \geqq n_{0}$ we have $x_{n} \notin T_{n_{0}}$. It follows that $x_{n} \notin S t\left(x, g_{N}\right)$ for $n \geqq n_{0}$, since $x_{n} \in Q$ for each $n$ and $Q \cap T_{n_{0}} \supset Q \cap S t\left(x, g_{N}\right)$. But this contradicts the convergence of the sequence $\left\{x_{n} \mid n=1,2, \cdots\right\}$ to $x$.

Hence we must have that there exists an integer $n_{0}$ such that $W_{n_{0}} \subset \overline{T_{n_{0}}}$, and the theorem is proved.

Definition. A space $X$ is collectionwise normal [4] if and only if for each discrete collection $S$ of subsets of $X$ there is a collection $S^{\prime}$ of mutually exclusive open sets covering $\cup\{D \mid D \in S\}$ such that no element of $S^{\prime}$ meets two elements of $S$.

In [14], McAuley proved that for semi-metric spaces collectionwise 
normality is equivalent to paracompactness.

COROLLARY 1.8. Every collectionwise normal locally separable $T_{0}$-space with a point-finite semi-devslopment is metrizable.

Proof. Such a space is locally metrizable by Theorem 1.7 and is paracompact by McAuley's result and Theorem 1.3. Therefore, it is metrizable by a theorem of Smirnov [17].

Proposition 1.9. Every Lindelof semi-developable space is separable.

Proof. Let $X$ be a Lindelof space with a semi-development $\Delta=\left\{g_{n} \mid n=1,2, \cdots\right\}$. For each integer $n$, consider the open cover of $X, S_{n}=\left\{\right.$ Int $\left.S t\left(x, g_{n}\right) \mid x \in X\right\}$. Since $X$ is a Lindelof space, there is a countable subset of $X, Q_{n}=\left\{y_{n}^{1}, y_{n}^{2}, \cdots\right\}$ such that $\Delta_{n}=\{\operatorname{Int}$ $\left.\operatorname{St}\left(y_{n}^{i}, g_{n}\right) \mid i=1,2, \cdots\right\}$ covers $X$. Let $Q=\cup\left\{Q_{n} \mid n=1,2, \cdots\right\}$. Then $Q$ is a countable subset which is dense in $X$. For suppose not, and let $x \in X \backslash \bar{Q}$. Then there is an integer $n$ such that $\operatorname{St}\left(x, g_{n}\right) \cap Q=\varnothing$. But $\Delta_{n}$ covers $X$, and so there is some $y_{n}^{i} \in Q_{n} \subset Q$ such that $x \in$ Int $S t\left(y_{n}^{i}, g_{n}\right) \subset S t\left(y_{n}^{i}, g_{n}\right)$. Hence $y_{n}^{i} \in S t\left(x, g_{n}\right) \cap Q$. Contradiction.

COROLLARY 1.10. Every regular Lindelof $T_{0}$-space with a pointfinite semi-development is a separable metric spase.

Proof. Theorem 1.7 and Proposition 1.9.

COROLlary 1.11. Every collectionwise normal locally Lindelof $T_{0}$-spase with a point-finite semi-development is metrizable.

Proof. Corollary 1.8 and Proposition 1.9.

Example 3.3 in [13] is an example of a separable semimetric space which is not hereditarily separable. The following proposition shows that this cannot happen in a point-finite s.d. space.

Proposition 1.12. A separable space with a point-finite semidevelopment is hereditarily separable.

Proof. Let $X$ be a space with a point-finite semi-development $\Delta=\left\{g_{n} \mid n=1,2, \cdots\right\}$ and a countable dense subset $Q$. Let $Y \subset X$. Define $S=\left\{G \mid G \in g_{n}\right.$ for some $\left.n, G \cap Q \neq \varnothing \neq G \cap Y\right\}$. Then, since $\Delta$ is point-finite and $Q$ is countable, $S$ is countable. For each $G \in S$ choose $p \in G \cap Y$ and let $P$ be the set of all points so chosen. Then 
$P$ is a countable subset of $Y$. Also $P$ is dense in $Y$. For let $U$ be an open set in $Y$ containing the point $y \in Y$. Let $g_{n}^{\prime}=\left\{G \cap Y \mid G \in g_{n}\right\}$ for each integer. Then $\left\{g_{n}^{\prime} \mid \imath=1,2, \cdots\right\}$ is a semi-development for $Y$, and therefore, there is an integer $n$ such that $y \in S t\left(y, g_{n}^{\prime}\right) \subset U$. Since $Q$ is dense in $X$, there is some $G \in g_{n}$ such that $G \cap Q \neq \varnothing$ and $y \in G$. Then $G \in S$, and hence, there is a point $p \in P$ contained in $G$, by definition of $P$. Therefore $p \in G \cap Y \subset S t\left(y, g_{n}^{\prime}\right) \subset U$, i.e, $U \cap P \neq \varnothing$. Hence, $y \in \bar{P}^{Y}$ which was to be proved.

It should be noted that this theorem is also true for pointcountable semi-developable spaces, by the same proof.

2. Some quotient images of metric spaces. We will consider in this section the images of metric spaces under certain kinds of maps. We will be primarily interested in pseudo-open maps. This class of functions was first defined by McDougle in [15] and has recently been rediscovered by Arhangel'skii [2] and Din' N'e T'ong [18]. $P$-maps vere defined by Ponomarev [16]. In [11], Heath proves that among $T_{1}$-spaces, the developable spaces are precisely the open $P$-images of metric spaces. The main results of this section are characterizations, among $T_{0}$-spaces, of pseudo-open $P$-images and pseudo-open compact images of metric spaces in terms of semi-developments. In addition, Int $P$-maps are defined, and, among $T_{0}$-spaces, the images of metric spaces under Int $P$-maps are shown to be the semi-developable spaces.

Throughout the remainder of this paper, by a map we shall mean a continuous function.

Definitions. Let $X$ and $Y$ be topological spaces, and let $f$ be a map from $X$ onto $Y$. In (2) and (3), let $X$ be a metric space.

(1) $f$ is pseudo-open if and only if for each $y \in Y$ and each open neighborhood $U$ of $f^{-1}(y)$ in $X, y \in \operatorname{Int} f(U)$.

(2) $f$ is a $P$-map if and only if for each $y \in Y$ and each open neighborhood $V$ of $y$, there is $\varepsilon>0$ such that $f\left[S\left(f^{-1}(y) ; \varepsilon\right)\right] \subset V$, where $S\left(f^{-1}(y) ; \varepsilon\right)=\left\{x \in X \mid d\left(x, f^{-1}(y)\right)<\varepsilon\right\}$.

(3) $f$ is an Int $P$-map if and only if $f$ is a $P$-map such that for each $\varepsilon>0$ and each $y \in Y, y \in \operatorname{Int} f\left[S\left(f^{-1}(y) ; \varepsilon\right)\right]$.

(4) $f$ is a compast map if and only if for each $y \in Y, f^{-1}(y)$ is a compact subset of $X$.

Note that $P$-map and Int $P$-map are metric and not topological properties. However, the definitions and the following related theorems may easily be restated topologically in terms of the existence of an appropriate metric.

A map $f$ from a space $X$ onto a space $Y$ is said to have a property hereditarily if and only if the restriction of $f$ to every 
saturated subset of $X$ has that property, i.e., for each $M \subset Y, f$ restricted to $f^{-1}(M)$ has the property.

THEOREM 2.1. Let $f$ be a map from the spase $X$ onto the space $Y$. Then the following conditions are equivalent.

(1) $f$ is pseudo-open.

(2) $f$ is hereditarily quotient.

(3) For each $M \subset Y, y \in \bar{M}$ if and only if $\left.f^{-1}(y) \cap \overline{f^{-1}(M}\right) \neq \varnothing$.

Proof. The equivalence of (1) and (2) is due to Arhangel'skii [2] and that of (1) and (3) is due to McDougle [15].

Lemma 2.2. Let $\Delta=\left\{g_{n} \mid n=1,2, \cdots\right\}$ be a semi-development for a $T_{0}$-space $X$. If $\left\{G_{n} \mid n=1, \cdots\right\}$ is a sequence of sets such that $G_{n} \in g_{n}$ for each $n$, then $\cap\left\{G_{n} \mid n=1,2, \cdots\right\}$ contains at most one point.

\section{Proof. Trivial.}

In the proofs of the subsequent theorems we need the following lemma. The proof of the lemma is routine and is omitted.

Lemma 2.3. Let $\left\{g_{n} \mid n=1,2, \cdots\right\}$ be a sequence of sets, and let $Z=\times\left\{g_{n} \mid n=1,2, \cdots\right\}=\left\{G=G_{1} G_{2} \cdots \mid G_{n} \in g_{n}\right.$ for each $\left.n\right\}$. Give $Z$ the product topology $p$ determined by giving each $g_{n}$ the discrete topology. Define $d: Z \times Z \rightarrow R$ as follows: For $G=G_{1} G_{2} \cdots, H=H_{1} H_{2} \cdots \in Z$, let $d(G, H)=1 / n$ where $n$ is the least integer such that $G_{n} \neq H_{n}$. Then $d$ defines a metric on $Z$ which is compatible with the product topology $p$ defined above.

Theorem 2.4. A To-space $Y$ is semi-developable if and only if it is the image of a metric space under an Int P-map.

Proof. Let $\Delta=\left\{g_{n} \mid n=1,2, \cdots\right\}$ be a refining semi-development for $Y$. Construct the metric space $Z$ from $\Delta$ as in Lemma 2.3. Let $X$ be the subspace of $Z$ consisting of all points $G=G_{1} G_{2} \cdots \in Z$ such that for some $y \in Y, \cap\left\{G_{n} \mid n=1,2, \cdots\right\}=\{y\}$. Let $d$ denote the metric on $X$ obtained by restricting the metric on $Z$ to $X$. Define a function $f$ from $X$ into $Y$ as follows: For $G=G_{1} G_{2} \cdots \in X$, let $f(G)=\cap\left\{G_{n} \mid n=1,2, \cdots\right\}$. Then $f$ takes $X$ onto $Y$ by Lemma 2.2. Also, $f$ is an Int $P$-map. For suppose $y \in Y$ and $U$ is an open neighborhood of $y$. Since $\Delta$ is a semi-development for $Y$, there is an integer $n$ such that $y \in \operatorname{Int} S t\left(y, g_{n}\right) \subset S t\left(y, g_{n}\right) \subset U$. Let 


$$
H=H_{1} H_{2} \cdots \in X
$$

be such that $d\left(H, f^{-1}(y)\right)<1 / n$. Then there is $G=G_{1} G_{2} \cdots \in X$ such that $f(G)=y$ and $d(G, H)<1 / n$, i.e., $G_{i}=H_{i}$ for $i=1, \cdots, n$. Hence, $y \in G_{i}=H_{i}$ for $i=1,2, \cdots, n$, and so

$$
f(H)=\cap\left\{H_{i} \mid i=1,2, \cdots\right\} \subset H_{n} \subset S t\left(y, g_{n}\right) \subset U .
$$

Thus, $f\left(S\left(f^{-1}(y) ; 1 / n\right)\right) \subset U$. It follows that $f$ is a $P$-map. If we show that for each integer $n$ and for each $y \in Y$,

$$
S t\left(y, g_{n}\right) \subset f\left(S\left(f^{-1}(y) ; 1 / n\right)\right),
$$

then clearly $f$ is an Int $P$-map. Let $n$ be any integer, let $y \in Y$, and let $z \in S t\left(y, g_{n}\right)$. Then there is $\mathrm{G}_{n} \in g_{n}$ such that $z, y \in G_{n}$. Hence, for $i=1,2, \cdots, n$, there is $G_{i} \in g_{i}$ such that $z, y \in G_{i}$, since $g_{i}<g_{i-1}$ for each $i$. Then choosing $H_{i} \in g_{i}$ such that $z \in H_{i}$ for each $i>n$ and letting $H_{i}=G_{i}$ for $i=1,2, \cdots, n$, we see that

$$
H=H_{1} H_{2} \cdots \in S\left(f^{-1}(y) ; 1 / n\right)
$$

and $f(H)=z$. Hence $z \in f\left(S\left(f^{-1}(y) ; 1 / n\right)\right)$. Therefore,

$$
S t\left(y, g_{n}\right) \subset f\left(S\left(f^{-1}(y) ; 1 / n\right)\right)
$$

which was to be shown.

Conversely, let $X$ be a metric space and let $f$ be an Int $P$-map from $X$ onto $Y$. For each integer $n$, let $g_{n}=\{f(S(x ; 1 / n)) \mid x \in X\}$. Then $J=\left\{g_{n} \mid n=1,2, \cdots\right\}$ is a semi-development for $Y$. For let $y \in Y$, and let $U$ be an open neighborhood of $y$. Since $f$ is an Int $P$-map, there is an integer $n$ such that $f\left(S\left(f^{-1}(y) ; 1 / n\right)\right)$ is a neighborhood of $y$ contained in $U$. Then $S t\left(y, g_{2 n}\right) \subset U$. For assume $y \in f(S(x ; 1 / 2 n))$ for some $x \in X$. Then $S(x ; 1 / 2 n) \cap f^{-1}(y) \neq \varnothing$, and therefore, $S(x ; 1 / 2 n) \subset S\left(f^{-1}(y) ; 1 / n\right)$. Hence,

$$
f(S(x ; 1 / 2 n)) \subset f\left(S\left(f^{-1}(y) ; 1 / n\right)\right) \subset U .
$$

Therefore, $S t\left(y, g_{2 n}\right) \subset U$. Finally for each integer $n$ and each $y \in Y$, $y \in \operatorname{Int} S t\left(y, g_{n}\right)$. This is so since for each integer $n$ and $y \in Y, y \in \operatorname{Int}$ $f\left(S\left(f^{-1}(y) ; 1 / n\right)\right)=\operatorname{Int}\left(\cup\left\{f(S(x ; 1 / n)) \mid x \in f^{-1}(y)\right\}\right) \subset \operatorname{Int} S t\left(y, g_{n}\right)$. Thus $\Delta$ is a semi-development for $Y$.

The same proof yields the following.

THEOREM 2.5. A $T_{0}$-space is semi-developable if and only if it is the image of a subspace of a zero-dimensional complete metric space under an Int P-map.

Remarks. An Int $P$-map need not be a quotient map as may be 
seen from the following example. Let $R=$ real numbers,

$$
X=\{(x, y) \in R \times R \mid 0<x \leqq 1 \text { and } y=1 / x \text {, or } x=0\},
$$

and $Y=\{x \in R \mid 0 \leqq x \leqq 1\}$. Let $X$ and $Y$ have the usual topologies, and let $X$ have the metric inherited from $R \times R$. Let $f$ be the projection from $X$ onto $Y$ defined by $f((x, y))=x$. Then $f$ is not a quotient map since $f^{-1}(A)$ is closed in $X$, but $A$ is not closed in $Y$, where $A=\{y \in Y \mid 0<y \leqq 1\}$. It is easy to see that $f$ is an Int $P$-map.

One important class of $P$-maps are the compact maps. If an Int $P$-map is a compact map, then it is also pseudo-open. Furthermore, every quotient Int $P$-map onto a $T_{2}$-space is a pseudo-open $P$-map. These statements are verified in Corollaries 2.10 and 2.11 below.

Theorem 2.6. A $T_{0}$-space is strongly semi-developable if and only if it is a pseudo-open P-image of a metric space.

Proof. Let $\Delta=\left\{g_{n} \mid n=1,2, \cdots\right\}$ be a strong refining semidevelopment for the $T_{0}$-space $X$. Define the metric space $X$ and the $P$-map $f$ from $X$ onto $Y$ as in Theorem 2.4. It remains to be shown that $f$ is pseudo-open. Let $M \subset Y$ and $y \in \bar{M}$. By Theorem 2.1, it suffices to show that $f^{-1}(y) \cap \overline{f^{-1}(M)} \neq \varnothing$. Now $y \in \bar{M}$ implies there is a descending sequence $\left\{G_{n} \mid n=1,2, \cdots\right\}$ of sets such that for each $n, y \in G_{n} \in g_{n}$ and $G_{n} \cap M \neq \varnothing$, since $\Delta$ is a strong semi-development. Thus $G=G_{1} G_{2} \cdots \in f^{-1}(y)$. Now let $\varepsilon>0$ and let $n$ be an integer such that $1 / n<\varepsilon$. Let $z \in G_{n} \cap M$. For $i>n$, we can choose $H_{i} \in g_{i}$ such that $z \in H_{i}$. For $i \leqq n$, let $H_{i}=G_{i}$. Then $\cap\left\{H_{i} \mid i=1,2, \cdots\right\}=z$, by Lemma 2 , and therefore, $H=H_{1} H_{2} \cdots \in X$. In particular, $H \in f^{-1}(M)$ since $z \in M$. Also $H_{i}=G_{i}$ for $i=1, \cdots, n$ implies $d(H, G) \leqq 1 / n<\varepsilon$. Hence we have shown that $S(G ; \varepsilon) \cap f^{-1}(M) \neq \varnothing$ for each $\varepsilon>0$. Thus $G \in \overline{f^{-1}(M)}$, and therefore, $G \in f^{-1}(y) \cap \overline{f^{-1}(M)}$.

Conversely, let $X$ be a metric space, and let $f$ be a pseudo-open $P$-map onto the $T_{0}$-space $Y$. For each integer $n$, let

$$
g_{n}=\{f(S(x ; 1 / n) \mid x \in X\},
$$

and let $\Delta=\left\{g_{n} \mid n=1,2, \cdots\right\}$. As was seen in Theorem 2.4, $\Delta$ is a semi-development for $Y$. There only remains to be shown that $\Delta$ is a strong semi-development for $Y$. Let $M \subset Y$, and $y \in \bar{M}$. Then $f$ is pseudo-open implies $f^{-1}(y) \cap \overline{f^{-1}(M)} \neq \varnothing$, by Theorem 2.1. Let $x \in f^{-1}(y) \cap \overline{f^{-1}(M)}$. Hence $S(x ; 1 / n) \cap f^{-1}(M) \neq \varnothing$ for each integer $n$ implies $f(S(x ; 1 / n)) \cap M \neq \varnothing$ for each integer $n$. Then letting $G_{n}=f(S(x ; 1 / n))$ for each integer $n$, we have that $\left\{G_{n} \mid n=1,2, \cdots\right\}$ 
is a descending sequence of sets such that for each $n, y \in G_{n} \in g_{n}$ and $G_{n} \cap M \neq \varnothing$. Hence, $\Delta$ is a strong semi-development for $Y$.

As before, the same proof yields.

Theorem 2.7. A $T_{0}$-space is strongly semi-developable if and only if it is the image of a subspace of a zero-dimensional complete metric space under a pseudo-open $P$-map.

Recall that a Fréchet space is a space $X$ which satisfies the following condition: For each $M \subset X, x \in \bar{M}$ if and only if there is a sequence in $M$ converging to $x$.

The following theorem is due to Arhangel'skii [2] and is proved by Franklin in [7].

THeOREM 2.8. If $X$ and $Y$ are Hausdorjf spaces, $X$ is a Fréchet spase, and $f$ is a quotiont map from $X$ onto $Y$, then $Y$ is a Frécht spase if and only if $f$ is pseudo-oper.

Theorem 2.9. Let $X$ be a metric space, $Y$ a $T_{2}$-space and $f a$ quotient P-map from $X$ onto $Y$. Then the following are equivalent:

(1) $Y$ is a Fréchet space.

(2) $Y$ is $1^{\text {st }}$ countable.

(3) $Y$ is semi-metrizable.

(4) $Y$ is strongly semi-metrizable.

(5) $f$ is pseudo-open.

Proof. (1) implies (5). Theorem 2.8, since metric spaces are Fréchet.

(5) implies (4). Theorem 1.8 and Theorem 2.6.

(4) implies ( 3 ). Immediate.

(3) implies (2). Immediate.

(2) implies (1). Well-known.

Corollary 2.10. Let $X$ be a metric space and $Y$ a $T_{2}$-spase. Then an Int P-map $f$ from $X$ onto $Y$ is a quotient map if and only if it is a pseudo-open P-map.

Corollary 2.11. Let $X$ be a metric space, $Y$ a space, and $f$ a compast Int $P$-map from $X$ onto $Y$. Then $f$ is pseudo-open.

Proof. Let $X, Y$ and $f$ be as stated, and let $U$ be an open set containing $f^{-1}(y)$, for some $y \in Y$. Then since $f^{-1}(y)$ is compact, there is $\varepsilon>0$ such that $S\left(f^{-1}(y) ; \varepsilon\right) \subset U$. Then, since $f$ is an Int $P$-map, $y \in$ Int $f\left(S\left(f^{-1}(y) ; \varepsilon\right)\right) \subset$ Int $f(U)$. 
THEOREM 2.12. A $T_{0}$-space is point-finite strongly semi-developable if and only if it is a pseudo-open compast image of a metris space.

Proof. Let $\Delta=\left\{g_{n} \mid n=1,2, \cdots\right\}$ be a refining point-finite strong semi-development for the $T_{0}$-space $Y$. Since $\Delta$ is a strong semidevelopment for $Y$, we can define a metric space $X$ and a pseudoopen map $f$ from $X$ onto $Y$ just as was done in the proof of Theorem 2.6. Hence the proof will be complete if it can be shown that $f$ is a compact map. Let $y \in Y$. For each integer $n$, let

$$
g_{n}(y)=\left\{G \in g_{n} \mid y \in G\right\} \text {. }
$$

Then, for each $n, g_{n}(y)$ is a finite set, by assumption. It is clear that for each sequence $G=G_{1} G_{1} \cdots$ such that $G_{n} \in g_{n}(y)$ for each $n$, we have that $\cap\left\{G_{n} \mid n=1,2, \cdots\right\}=\{y\}$, i.e., $G \in X$ and, in particular, $G \in f^{-1}(y)$. Hence it is clear that $f^{-1}(y)=\left\{G=G_{1} G_{2} \cdots \mid G_{n} \in g_{n}(y)\right\}$. By Lemma 2.3, the metric topology on $X$ is compatible with the subspace topology of $Z=\times\left\{g_{n} \mid n=1,2, \cdots\right\}$ where each $g_{n}$ is given the discrete topology and $Z$ is given the product topology. Hence we have that $f^{-1}(y)=\times\left\{g_{n}(y) \mid n=1,2, \cdots\right\}$ is compact, being the product of compact sets, since each $g_{n}(y)$ is a finite discrete set. Thus $f$ is a compact map.

Conversely, let $X$ be a metric space, and let $f$ be a pseudo-open compact map from $X$ onto $Y$. For each positive integer $n$, let $\mathscr{A}_{n}$ be an open cover of $X$ which is a locally finite refinement of $\{S(x ; 1 / n) \mid x \in X\}$. This can be done since $X$ is a paracompact space. Let $\mathscr{B}_{1}=\mathscr{A}_{1}$, and for $n>1$, let $\mathscr{B}_{n}=\mathscr{B}_{n-1} \wedge \mathscr{A}_{n}$. For each positive integer $n$, let $g_{n}=\left\{f(B) \mid B \in \mathscr{B}_{n}\right\}$, and let $\Delta=\left\{g_{n} \mid n=1,2, \cdots\right\}$. Then $\Delta$ is a semi-development. For let $U$ be an open neighborhood of a point $y \in Y$. Since $f$ is a compact map and consequently a $P$-map, there is an integer $n$ such that $f\left(S\left(f^{-1}(y) ; 1 / n\right)\right) \subset U$. Then $S t\left(y, g_{2 n}\right) \subset U$. For let $y \in f(B)$ where $B \in \mathscr{\mathscr { S }}_{2 n}$. By definition of $\mathscr{S}_{2 n}$, there is $x \in X$ such that $B \subset S(x ; 1 / 2 n)$. Then $y \in f(S(x ; 1 / 2 n))$; so

$$
S(x ; 1 / 2 n) \cap f^{-1}(y) \neq \varnothing,
$$

and therefore, $S(x ; 1 / 2 n) \subset S\left(f^{-1}(y) ; 1 / n\right)$. Hence,

$$
f(B) \subset f(S(x ; 1 / 2)) \subset f\left(S\left(f^{-1}(y) ; 1 / n\right)\right) \subset U .
$$

Thus, $S t\left(y, g_{2 n}\right) \subset U$. Now for each $y \in Y$ and each integer $n$, let $\mathscr{B}_{n}(y)=\left\{B \in \mathscr{\mathscr { P }}_{n} \mid f^{-1}\{y) \cap B \neq \varnothing\right\}$. Then $\mathscr{\mathscr { B }}_{n}(y)=\left\{B \in \mathscr{B}_{n} \mid y \in f(B)\right\}$. Hence, $\cup\left\{f(B) \mid B \in \mathscr{\mathscr { S }}_{n}(y)\right\}=S t\left(y, g_{n}\right)$. Now $\cup\left\{B \mid B \in \mathscr{\mathscr { B }}_{n}(y)\right\}$ is an open set containing $f^{-1}(y)$ and $f$ is pseudo-open, and so

$$
y \in \operatorname{Int} f\left(\cup\left\{B \mid B \in \mathscr{\mathscr { S }}_{n}(y)\right\}\right)=\operatorname{Int}\left(\cup\left\{f(B) \mid B \in \mathscr{\mathscr { S }}_{n}(y)\right\}\right)=\operatorname{Int} \operatorname{St}\left(y, g_{n}\right) .
$$


Thus we have shown that $\Delta$ is a semi-development for $Y$.

$\Delta$ is also a strong semi-development for $Y$. First note that for any $H \subset X, x \in \bar{H}$ implies there exists a descending sequence of sets $\left\{B_{n} \mid n=1,2, \cdots\right\}$ such that for each $n, x \in B_{n} \in \mathscr{B}_{n}$ and $B_{n} \cap H \neq \varnothing$. This is true because each $\mathscr{A}_{n}$ is an open cover and, by construction, $\mathscr{B}_{n}=\mathscr{A}_{1} \wedge \cdots \wedge \mathscr{A}_{n}$, for each $n$. Now assume $M \subset Y$ and $y \in \bar{M}$. Since $f$ is pseudo-open we have $f^{-1}(y) \cap \overline{f^{-1}(M)} \neq \varnothing$. So let

$$
x \in f^{-1}(y) \cap \overline{f^{-1}(M)} .
$$

Then $x \in \overline{f^{-1}(M)}$ implies, by the comment above, that there is a descending sequence of sets $\left\{B_{n} \mid n=1,2, \cdots\right\}$ such that for each $n$, $x \in B_{n} \in \mathscr{B}_{n}$ and $B_{n} \cap f^{-1}(M) \neq \varnothing$. Then $\left\{f\left(B_{n}\right) \mid n=1,2, \cdots\right\}$ is a descending sequence of sets such that $f\left(B_{n}\right) \cap M \neq \varnothing$ and $f\left(B_{n}\right) \in g_{n}$ for each $n$. Also, $x \in f^{-1}(y) \cap B_{n}$ for each $n$, and hence, $y \in f\left(B_{n}\right)$ for each $n$. Thus $\Delta$ is a strong semi-development for $Y$.

Finally $\Delta$ is point-finite. For let $y \in Y$ and let $n$ be any integer. $\mathscr{B}_{n}$ is locally finite and $f^{-1}(y)$ is compact. Therefore, there is an open set containing $f^{-1}(y)$ which meets only finitely many members of $\mathscr{B}_{n}$. Hence $f^{-1}(y) \cap B \neq \varnothing$ for only finitely many $B \in \mathscr{P}_{n}$, and therefore, $y \in f(B)$ for only finitely many $B \in \mathscr{S}_{n}$.

As before, the same proof yields

THEOREM 2.13. A $T_{0}$-space is point-finite strongly semi-developable if and only if it is the image of a subspace of a zero-dimensional complete metric space under a pseudo-open compact map.

CoRollary 2.14. Let $X$ be a regular separable $T_{0}$-space which is a pseudo-open compact image of a metric space. Then $X$ is metrizable.

\section{Proof. Theorem 1.7 and Theorem 2.12 above.}

CoRollary 2.15. [3; page 128]. Let $X$ be a regular Lindelof $T_{0}$-space which is a pseudo-open compact image of a metric space. Then $X$ is metrizable.

Proof. Proposition 1.9 and Corollary 2.14 above.

Arhangel'skii has asked the following question: Is a paracompact $T_{2}$-space that can be expressed as a pseudo-open compact image of a metric space metrizable?

Corollary 2.15 answers a weakened version of his question affirmatively, and it is thought that Theorem 2.12 may aid in the eventual resolution of the problem. 
TheOREM 2.16. A collectionwise normal $T_{0}$-space $Y$ is metric and locally separable if and only if $Y$ is a pseudo-open compast image of a metric locally separable space.

Proof. The necessity of the condition is immediate. For the sufficiency, let $X$ be a metric locally separable space, and let $f$ be a pseudo-open compact map from $X$ onto $Y$. By Theorem 2.12 and Corollary 1.8, it suffices to show that $Y$ is locally separable. Let $y \in Y$. Now $X$ is locally separable implies that for each $x \in f^{-1}(y)$ there is an open separable subset of $X, U(x)$, containing $x$. Since $f^{-1}(y)$ is compact there exist $x_{1}, \cdots, x_{n} \in f^{-1}(y)$ such that

$$
f^{-1}(y) \subset U=\cup\left\{U\left(x_{i}\right) \mid i=1, \cdots, n\right\} .
$$

Then $U$ is clearly separable, and hence, so is $f(U)$ since $f$ is continuous. Also $U$ is an open set containing $f^{-1}(y)$, and therefore, $f(U)$ is a neighborhood of $y$, since $f$ is pseudo-open. Thus, since separability is hereditary in a $T_{0}$-space with a point-finite semidevelopment, by Proposition 1.12, $Y$ is locally separable.

THeorem 2.17. A collestionwise normal $T_{0}$-space $Y$ is a locally compast mstris spase if and only if $Y$ is a psəudo-spon compact image of a locally compact motris spasc.

Proof. The necessity is trivial. Note, by Proposition 1.9, that a locally compact $T_{0}$-space with a point-finite semi-development is locally separable. Therefore it is metrizable, by Corollary 1.8, if it is collectionwise normal. Hence it suffices to show that local compactness is preserved by pseudo-open compact maps. An argument analogous to that in the previous theorem shows this.

\section{REFERENCES}

1. P. S. Alexandrov and V. V. Niemytskii, Metrizability conditions for topological spaces and the axiom of symmetry, Mat. Sb. 3 (1938), 663-672.

2. A. V. Arhangel'skii, Some types of factor mappings, and the relations between classes of topological spaces, Dokl. Akad. Nauk SSSR 153 (1963), 743-746; Soviet Math. Dokl. 4 (1963), 1726-1729.

3. - Mappings and spaces, Uspekhi Mat. Nauk. 21 (1966), 133; Russian Math. Surveys 21 (1966), 115-162.

4. R. H. Bing, Metrization of topological spaces, Canadian J. Math., 3 (1951), 175-186.

5. Morton Brown, Semi-metric spaces, Summer Institute on Set Theoretic Topology, Madison, Wisconsin, Amer. Math. Soc., (1955), 62-64.

6. J. G. Ceder, Some generalizations of metric spaces, Pacific J. Math., 11 (1961), $105-126$.

7. S. P. Franklin, Spaces in which sequences suffice, Fund. Math., 57 (1965), 107-115.

8. A. H. Frink, Distance functions and the metrization problem, Bull. Amer. Math. Soc., 43 (1937), 133-142. 
9. R. W. Heath, A regular semi-metric space for which there is no semi-metric under which all spheres are open, Proc. Amer. Math. Soc., 12 (1961), 810-811.

10. - Arc-wise connectedness in semi-metric spaces, Pacific J. Math., 12 (1962), 1301-1319.

11. - On open mappings and certain spaces satisfying the first countability axiom, Fund. Math., 57 (1965), 91-96.

12. J. L. Kelley, General Topology, Van Nostrand, Princeton, N. J., 1955.

13. L. F. McAuley, A relation between perfect separability, completeness and normality in semi-metric spaces, Pacific J. Math., 6 (1956), 315-326.

14. L. F. McAuley, A note on complete collectionwise normality and paracompactness, Proc. Amer. Math. Soc., 9 (1958), 796-799.

15. P. McDougle, A theorem on quasi-compact mappings, Proc. Amer. Math. Soc., 9 (1958), 474-477.

16. V. I. Ponomarev, Axioms of countability and continuous mappings, Bull. Pol. Akad. Nauk., 8 (1960), 127-134.

17. Yu. M. Smirnov, On metrization of topological spaces, Uspekhi Mat. Nauk. 6 (1951); Amer. Math. Soc. Translation no. 91 (1953).

18. Din' N' e T'ong, Preclosed mappings and Taimanov's theorem, Dokl. Akad, Nauk. SSSR 152 (1963) 525-528; Soviet Math. Dokl. 4. (1963), 1335-1338.

Received June 30, 1939 and in revised form September 21, 1970. This paper was written while the author was supported by National Aeronautics and Space Administration Traineeship Grant NFG (T) 55-2. The author wishes to thank Professor C. J. Himmelberg for his advice and encouragement.

UNIVERSITY OF VIRGINIA 



\section{PACIFIC JOURNAL OF MATHEMATICS}

\section{EDITORS}

\author{
H. SAMElson \\ Stanford University \\ Stanford, California 94305 \\ C. R. HOBBY \\ University of Washington \\ Seattle, Washington 98105
}

J. DugundjI

Department of Mathematics University of Southern California Los Angeles, California 90007

RICHARD ARENS

University of California

Los Angeles, California 90024

\section{ASSOCIATE EDITORS}
E. F. BECKENBACH
B. H. NeUmanN
F. WOLE
K. YOSHIDA

\section{SUPPORTING INSTITUTIONS}

\author{
UNIVERSITY OF BRITISH COLUMBIA \\ CALIFORNIA INSTITUTE OF TECHNOLOGY \\ UNIVERSITY OF CALIFORNIA \\ MONTANA STATE UNIVERSITY \\ UNIVERSITY OF NEVADA \\ NEW MEXICO STATE UNIVERSITY \\ OREGON STATE UNIVERSITY \\ UNIVERSITY OF OREGON \\ OSAKA UNIVERSITY \\ UNIVERSITY OF SOUTHERN CALIFORNIA
}

\author{
STANFORD UNIVERSITY \\ UNIVERSITY OF TOKYO \\ UNIVERSITY OF UTAH \\ WASHINGTON STATE UNIVERSITY \\ UNIVERSITY OF WASHINGTON \\ * * * \\ AMERICAN MATHEMATICAL SOCIETY \\ CHEVRON RESEARCH CORPORATION \\ NAVAL WEAPONS CENTER
}

The Supporting Institutions listed above contribute to the cost of publication of this Journal, but they are not owners or publishers and have no responsibility for its content or policies.

Mathematical papers intended for publication in the Pacific Journal of Mathematics should be in typed form or offset-reproduced, (not dittoed), double spaced with large margins. Underline Greek letters in red, German in green, and script in blue. The first paragraph or two must be capable of being used separately as a synopsis of the entire paper. The editorial "we" must not be used in the synopsis, and items of the bibliography should not be cited there unless absolutely necessary, in which case they must be identified by author and Journal, rather than by item number. Manuscripts, in duplicate if possible, may be sent to any one of the four editors. Please classify according to the scheme of Math. Rev. Index to Vol. 39. All other communications to the editors should be addressed to the managing editor, Richard Arens, University of California, Los Angeles, California, 90024.

50 reprints are provided free for each article; additional copies may be obtained at cost in multiples of 50 .

The Pacific Journal of Mathematics is published monthly. Effective with Volume 16 the price per volume (3 numbers) is $\$ 8.00$; single issues, $\$ 3.00$. Special price for current issues to individual faculty members of supporting institutions and to individual members of the American Mathematical Society: $\$ 4.00$ per volume; single issues $\$ 1.50$. Back numbers are available.

Subscriptions, orders for back numbers, and changes of address should be sent to Pacific Journal of Mathematics, 103 Highland Boulevard, Berkeley, California, 94708.

PUBLISHED BY PACIFIC JOURNAL OF MATHEMATICS, A NON-PROFIT CORPORATION

Printed at Kokusai Bunken Insatsusha (International Academic Printing Co., Ltd.), 7-17, Fujimi 2-chome, Chiyoda-ku, Tokyo, Japan. 


\section{Pacific Journal of Mathematics}

\section{Vol. 37, No. $2 \quad$ February, 1971}

Charles Compton Alexander, Semi-developable spaces and quotient images of metric spaces .................................... 277

Ram Prakash Bambah and Alan C. Woods, On a problem of Danzer. . . . . . . . . 295

John A. Beekman and Ralph A. Kallman, Gaussian Markov expectations and related integral equations . ....................................

Frank Michael Cholewinski and Deborah Tepper Haimo, Inversion of the Hankel

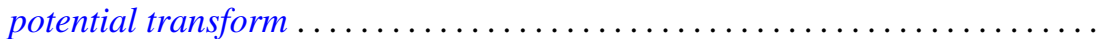

John H. E. Cohn, The diophantine equation

$$
Y(Y+1)(Y+2)(Y+3)=2 X(X+1)(X+2)(X+3) \ldots \ldots \ldots \ldots \ldots
$$

Philip C. Curtis, Jr. and Henrik Stetkaer, A factorization theorem for analytic

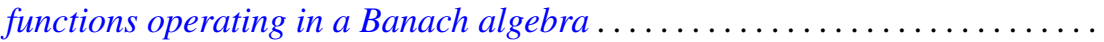

Doyle Otis Cutler and Paul F. Dubois, Generalized final rank for arbitrary limit ordinals

Keith A. Ekblaw, The functions of bounded index as a subspace of a space of entire functions

Dennis Michael Girard, The asymptotic behavior of norms of powers of

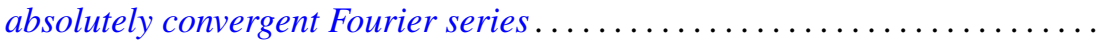

John Gregory, An approximation theory for elliptic quadratic forms on Hilbert spaces: Application to the eigenvalue problem for compact quadratic forms. 383

Paul C. Kainen, Universal coefficient theorems for generalized homology and stable cohomotopy.

Aldo Joram Lazar and James Ronald Retherford, Nuclear spaces, Schauder bases, and Choquet simplexes.

David Lowell Lovelady, Algebraic structure for a set of nonlinear integral operations

John McDonald, Compact convex sets with the equal support property . 429

Forrest Miller, Quasivector topologies

Marion Edward Moore and Arthur Steger, Some results on completability in commutative rings.

A. P. Morse, Taylor's theorem

Richard E. Phillips, Derek J. S. Robinson and James Edward Roseblade, Maximal subgroups and chief factors of certain generalized soluble groups.

Doron Ravdin, On extensions of homeomorphisms to homeomorphisms ...

John William Rosenthal, Relations not determining the structure of $\mathrm{L}$

Prem Lal Sharma, Proximity bases and subbases ........... .

Larry Smith, On ideals in $\Omega_{*}^{u}$. .

Warren R. Wogen, von Neumann algebras generated by operators similar to normal operators

R. Grant Woods, Co-absolutes of remainders of Stone-Čech 